\title{
Desktop Virtualization with Cloud Computing
}

\author{
${ }^{\#}$ Charul Jagtap, "Shweta Patange, "Ritesh Mahajan, "Mamta Fasge, Prof. Santosh Waghmode" \\ \#Student, "Professor, \\ Department of Computer Science, \\ JSPM's Imperial College of Engineering and Research, Pune \\ Savitribai Phule Pune University \\ Pune, Maharashtra, India
}

\begin{abstract}
In today's era Desktop Virtualization is depreciated and is no longer used in the corporate world and institutions. One of the major problems we face these days is of storage and security issue which can be resolved with the integration of cloud computing. In this paper, we have integrated Cloud Computing with Desktop Virtualization. In our research and project, we have deployed a cloud using openstack along with Desktop Virtualization. This paper aims for the security and cost reduction for institutional purpose.
\end{abstract}

Keywords:- Cloud Computing, Desktop Virtualization, Openstack.

\section{I.INTRODUCTION}

As IT sectors and Institutions are expanding rapidly day by day, need for computer systems are also increased. new machines are being purchased. The IT companies can easily afford buying new machines as per their requirements. But what about the institutions and educational sectors? Hence an effective way should be determined for the usage. N-Computing i.e. Desktop Virtualization with Cloud provides us with the solution. It helps to resolve many concerns such as Cost reduction, Data Security and Efficient use of storage and technology. Cloud Computing helps to provide maximum storage with low computational cost. Hence the Institutions find this method more affordable and promising. which reduces the total hardware with software.Enabling technology of cloud computing, virtualization provides a realistic solution for resource consolidation and simplifying the management. On the other hand it helps an organization to build a green computing environment with the increasing popularity of cloud computing with virtualization technology. By using software as a service(SAAS), users can access software and database in the business model depending on their own sectors. The management of infrastructure and platform is done using cloud providers. Sometimes, software as a service is referred to as ondemand software because of the cloud providers charge on- pay per use basis for accessing the different services provided by them. So this causes proponents to get the computing resources in outsourcing manner. That's why the maintenance of hardware and software is being reduced for users. Introduction of cloud computing avoids direct management of hardware and it causes great convenience to users. It also reduces the maintenance cost of these hardwares. Desktop Virtualization provides new extension of virtualization technology which has prominent advantages such as centralized management, securing the data and reducing overall cost. According to the study of current methodology, we noticed that replacing number of CPUs with the other computational devices or thin clients is not a promising solution. It only reduces the cost and size of the hardware used but also has the limitations at user end. Every system requires its own processing device. without this thin client none of the system can work, hence we have taken the initiative to replace those computing devices with cloud which provides computational power to those systems. At Present, each Desktop is provided with the dedicated CPU and all the work is carried out. But in our work, we have used only one computing source, that is a server to provide computational power to 3 different machines. This model consists of a Raspberry Pi configured with Virtualization and Cloud Computing as shown in Fig.1.

Desktop Virtualization is a technique that separates computing environment from physical client device. Here in N-computing, only one server provides computational power to other desktops. The other monitors need not have their own computing power i.e. CPU. Each monitor will act as an independent machine with an Operating System. Our project and study aims for the usage of single computational unit serving to three different workstations with the scope of reducing the computational cost and efficient use of cloud storage. The cloud storage here is provided with the deployment of Openstack platform. All of the activities are carried on Raspberry Pi model 4, depending upon the hardware and software compatibility. Since project is carried on Raspberry Pi, specific operating systems are only supported by it. Here for our project and research, we have selected ubuntu operating system. The aim of this project is to increase the performance of the model which can be obtained by using the Operating Systems which having fewer RAM utilization with less CPU response time and high application response time. As Linux provides great performance ubuntu OS is used. For Cloud deployment, Openstack platform is used. OpenStack is an open source platform that uses pooled virtual resources to build and manage private and public clouds. The tools that comprise the OpenStack platform, called "projects", handle the core cloud-computing services of compute, networking, storage, identity, and image services. Nova is the primary computing engine behind OpenStack. It is used for deploying and managing large numbers of virtual machines and other instances to handle the computing tasks. It helps to ensure that 
each of the components of an OpenStack deployment can communicate with one another quickly and efficiently.

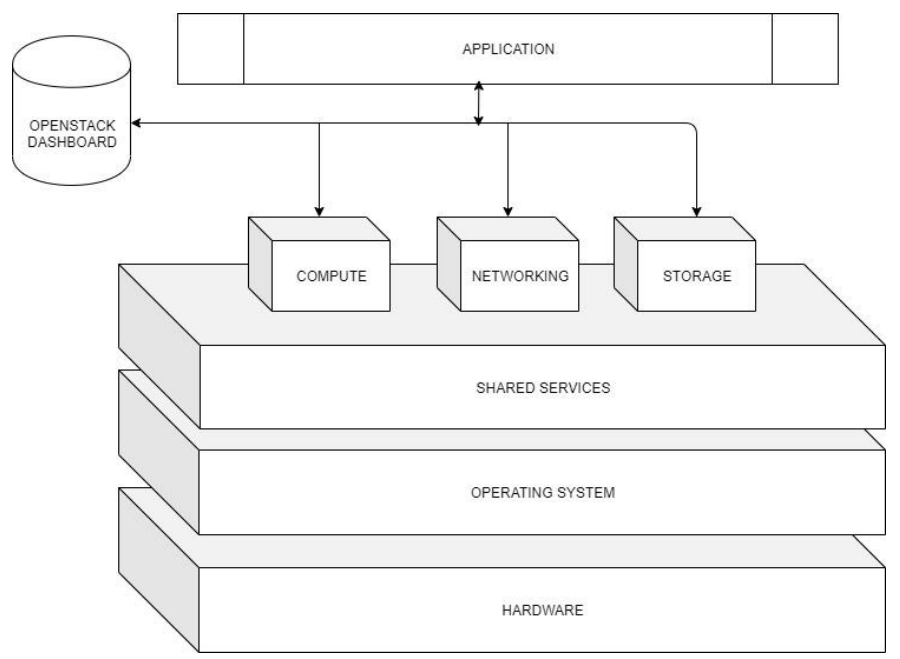

Fig.1.1 Openstack Architecture

\section{TRADITIONAL WAY AND PROBLEMS}

Traditionally, N-Computing was achieved by means of $\mathrm{N}$ computing devices and virtualization software work by distributing the computing power of a desktop PC among several workstations. This method consists of a hardware namely Thin Client and which is responsible for N-computing. The unique N-Computing technology is composed of three primary components: vSpace virtualization software, a user extension protocol, and access devices. By combining all three of these components into an integrated solution, N-Computing delivers unmatched performance at an incredible low cost. We found out that there was an issue on the use of Thin Client particularly in the multimedia application and on the issue of the user acceptance of Thin Client.

Basically, this vSpace device is connected to monitor and then it acts as an independent machine. The vSpace device acts as a Central Processing Unit. which takes the processing power from the main CPU server But the device lacks cloud computing. This device is meant for small scale use only. One vSpace device can be connected to only one monitor. Hence, the major issues that these devices face is of integration with Cloud Computing and ability to offer virtualization to multiple monitors. Another issue is the selection of the type and model of N-Computing product which plays an important role that affects the performance of the technology. Moreover, earlier versions and models of $\mathrm{N}$-Computing devices seem to be slower compared to the new ones. We noticed that, each computer system requires its own separate hardware resources with leads to high costing as well as high maintenance. Hence it's a good idea to replace $\mathrm{H} / \mathrm{W}$ resources with $\mathrm{S} / \mathrm{W}$ resources. The model based on current methodologies consists of various drawbacks based on performance,speed,storage, security and multimedia usage.

\section{PROPOSED METHODOLOGY}

Proposed methodology consists of 2 Raspberry Pi model 4 (cluster of Raspberry Pi) configured together to provide cloud storage and desktop virtualization. The concept and scope of this paper is to boot up three systems with the help of only a single computational device without using any extra external hardware to provide the processing power. For cloud storage, additional 1TB external hard drive is provided. Firstly, a suitable operating system is installed and booted on the Raspberry Pi using a bootable pendrive. preferably linux based OS. Once the OS is installed, virtualization tools software like VMware is installed and enabled. Later, cloud is deployed using Openstack platform. The Raspberry Pi will be attached with an external 1 TB HDD with 4 GB of swap memory and LVM (Logical Volume Management). Later, three 3 LCD monitors are attached to this Raspberry Pi and is made to boot up with OS on each monitor with the help of VMware. Each workstation will be allocated a $100 \mathrm{~GB}$ of local storage and 500 GB memory will be used as cloud storage. These three LCD monitors will then act as an independent workstations. The users can store the organizational data on these local storages of $100 \mathrm{~GB}$ each. The sensitive data can be stored on cloud. which increases the user privacy and data security.

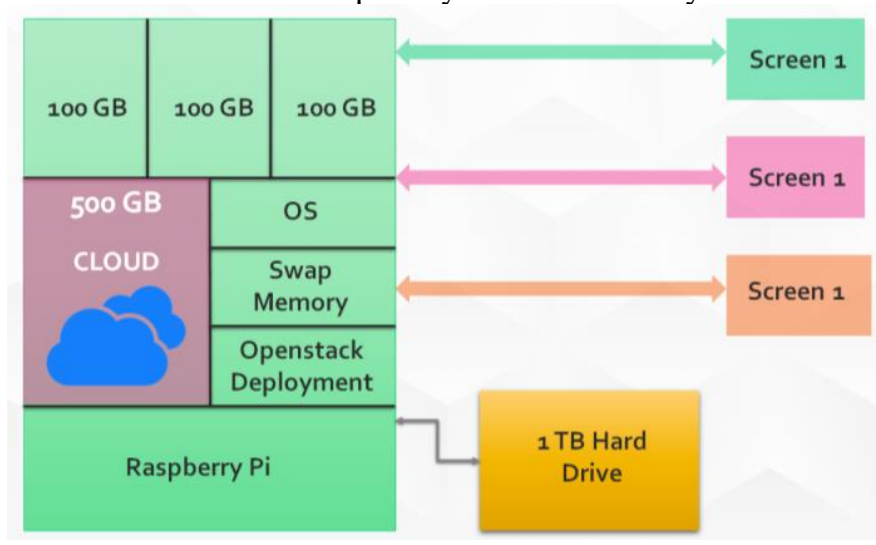

Fig.1.2 Desktop Virtualization Architecture

The gap between the proposed model and the traditional model is filled and overcome with virtualizing multiple workstations at a time and provide the cloud storage. The proposed model accounts for better utilization of computational power and storage with minimum costing. This model is based on a linux system in which virtualization will be enabled. This architecture can support three workstations efficiently. We have chosen linux based operating system as they require less amount of RAM and less computational power. A basic linux machine running ubuntu 16.04 require $2 \mathrm{~GB}$ of RAM. Thus this model with the help of SWAP memory and RAM will provide computational power to 3 machines. Basically this architecture will act as a server and other three workstations will act as client nodes. The two raspberry pi can be connected with each other to avail maximum performance. one can add more number of raspberry pi based on the need. This is done by executing the following steps:

1. Connecting to the RPi (Raspberry Pi) through IP address.

2. Building a LXC Container (Linux Container)

3. Launching LXC Container.

4. Mounting cgroup

5. Creating a directory to store Hosts.

6. Creating a file system for the container.

7. Changing root privileges. 


\begin{tabular}{|c|c|c|c|}
\hline \multicolumn{4}{|c|}{ IV. ARCHITECTURE DETAILS } \\
\hline \multirow{2}{*}{$\begin{array}{l}\text { Sr. } \\
\text { No }\end{array}$} & \multicolumn{3}{|c|}{ ARCHITECTURE DETAILS } \\
\hline & $\begin{array}{l}\text { Components/ } \\
\text { Partitions }\end{array}$ & Size/ version & Purpose \\
\hline [1] & Raspberry-Pi & Model4 & $\begin{array}{l}\text { Computational } \\
\text { Device }\end{array}$ \\
\hline [2] & External HardDrive & $1 \mathrm{~TB}$ & Storage \\
\hline [3] & Workstations x3 & $\begin{array}{l}\text { 5'(inch) } \\
\text { or Desktop } \\
\text { Monitors }\end{array}$ & $\begin{array}{l}\text { Virtualized } \\
\text { Independent } \\
\text { Machines }\end{array}$ \\
\hline [4] & Operating System & $\begin{array}{l}\text { Ubuntu } \\
16.04\end{array}$ & Interface \\
\hline [5] & Swap Memory & 4 GB & Performance \\
\hline [6] & HDD Partitions x3 & $100 \mathrm{~GB}$ & $\begin{array}{l}\text { Independent Storage } \\
\text { for Workstations }\end{array}$ \\
\hline [7] & Openstack & $500 \mathrm{~GB}$ & Cloud Deployment \\
\hline
\end{tabular}

Fig. 2 Table 1

The above table represented overview of all the components comprised in this architecture along with its storage segregation and allocation. Many additional packages, repositories need to be installed as each operating system has a different kernel and different dependencies. Also, along with the installation of packages and repositories, we have to enable the specific services and daemons needed for the functioning of openstack and virtualization. Rest the other unnecessary services and daemons need to be disabled. Also iptables and firewall does not work simultaneously. As both the commands does the same thing collision may occur. Thus we need to enable only the firewalld and disable the iptables. Also, mask the services to nullify the effect of accidental invoke of these services. Once the iptable services are disabled and masked the firewall will have no interference while carrying out the openstack deployment.

\section{FLOWCHART}

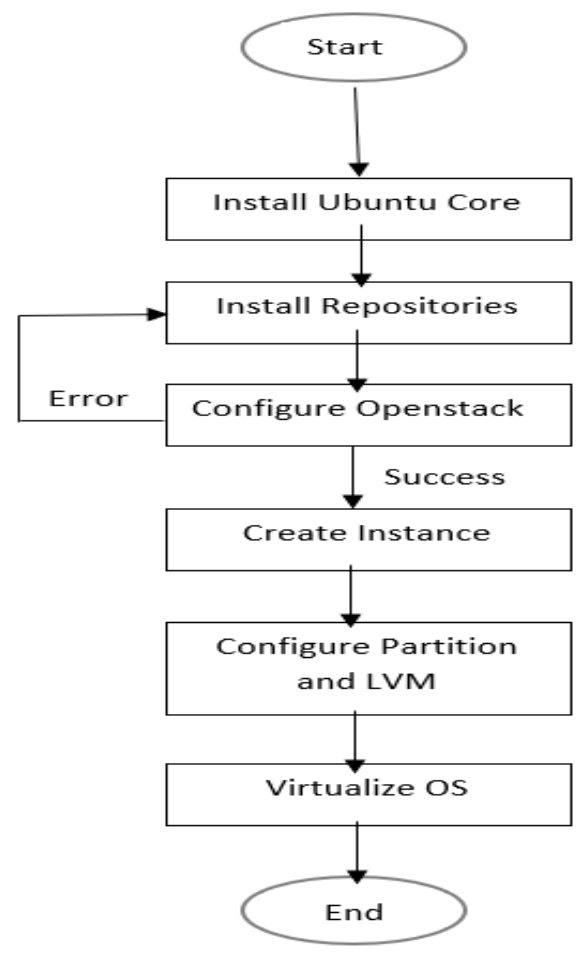

Fig. 3 System Workflow

\section{ADVANTAGES}

1. Replacing hardware with software which results into low costing and maintenance.

2. Servers and thin clients cost less to acquire than an army of PCs.

3. Thin clients use less energy.

4. Generate a fraction of a PCs fan noise and heat.

5. Greatly reduce the need for hands-on support.

6. Even use existing PCs past their expiration dates via software clients.

\section{CONCLUSION}

Hence, the integration of this depreciated technology with the exalting technology leads to the upgrading product. A model comprising of Desktop Virtualization and Cloud Computing at a low computational cost is achieved for the betterment and efficient use of storage and technology. This model can be very useful in the educational sector and at the institute level. This model can be deployed and used in college practical labs, in account section and various departments for daily use.

\section{REFERENCES}

[1] Sunwook Kim and jihyeok Choi, "Cloud-based Virtual Desktop Service Using Lightweight Network Display Protocol". Daejeon,Republic of Korea, IEEE-2016.

[2] Xi Liu, Mingfa Zhu, Limin Xiao and Yuanjie Jiang, “ A VM-Shared Desktop VirtualiZation System Based on Openstack", Beihang,China.

[3] P.H. Nakhai and N.B. Anuar " Performance Evaluation of Virtual Desktop Operating Systems in Virtual Desktop Infrastructure", Kuala Lumpur, Malaysia, IEEE-2017.

[4] Esmael V. Maliberan, " N-Computing Adoption: Issues and Challenges - International Journal(2019)" Rosario, Tandag City2019. 